\title{
Amadeus
}

International Multidisciplinary Journal IISN 2525-8281

DOI: 10.14295/aimj.v4i7.82

\section{Heart Failure Predictors in Adult Population}

Luiz Arthur Bevilaqua Bandeira $^{l}$;

Talina Carla da Silva

Macerlane Lira Silva ${ }^{3}$; Elisangela Vilar de Assis Melina Maria Loiola Melo

Vasconcelos ${ }^{5}$.

Ana Rebeca Soares Maia de

Oliveira $^{6}$

\begin{abstract}
Cardiovascular insufficiency (HF) has been shown to cause morbidity and mortality in several countries, among other aspects, due to changes in habits and lifestyle, leading to an increase in life expectancy. It is considered a major public health problem given the high prevalence in the adult population, especially in the age group above 65 years. The present study discusses the predictors of HF, based on an integrative review of four studies on the subject. The results showed that, among the risk factors that most tend to imply the onset of $\mathrm{HF}$, are systemic arterial hypertension, diabetes, coronary artery disease, obesity, dyslipidemia, and other factors such as smoking, gender and age.
\end{abstract}

Keywords: heart failure, public health, adult population.

\section{Preditores de Insuficiência Cardíaca na População Adulta}

\begin{abstract}
Resumo: A insuficiência cardiovascular (IC), tem se mostrado causa de morbimortalidade em diversos países, dentre outros aspectos, por conta de mudanças de hábitos e estilo de vida, implicando em um aumento na expectativa de vida. É considerada um grande problema de saúde pública ante a alta prevalência na população adulta, principalmente na faixa etária acima de 65 anos. O presente estudo discute os preditores da IC, à partir de uma revisão integrativa de quatro estudos sobre a temática. Os resultados demonstraram que, entre os fatores de risco que mais tendem a implicar no surgimento da IC, são a hipertensão arterial sistêmica, diabetes, doença da artéria coronária, obesidade, dislipidemias, além de outros fatores, como tabagismo, sexo e idade.
\end{abstract}

Palavras-chave: insuficiência cardíaca, saúde pública, população adulta.

\footnotetext{
${ }^{1}$ Acadêmico de Medicina da Faculdade Santa Maria de Cajazeiras, PB. arthurbevilaqua@ hotmail.com;

${ }^{2}$ Graduação em Enfermagem pela Faculdade Santa Maria de Cajazeiras, Especialização em Política e Gestão do Cuidado pela UFPB e Mestrado em Saúde Coletiva pela UNISANTOS. Presidente da Associação de Pais e Amigos dos Excepcionais de Cajazeiras , Brasil

${ }^{3}$ Acadêmica de Medicina da Faculdade Santa Maria de Cajazeiras, Paraíba, Brasil;

${ }^{4}$ Doutorado em Ciências da Saúde pela Faculdade de Medicina do ABC_SP. Mestre em Ciências da Nutrição pela Universidade Federal da Paraíba, Graduada em Fisioterapia pelo Centro Universitário de João Pessoa. Professora da Universidade Federal de Campina Grande, Paraíba, Brasil;

${ }^{5}$ Acadêmica de Medicina pela UNICHRISTUS, Ceará, Brasil;

${ }^{6}$ Graduação em Medicina pela Universidade Federal do Ceará, Brasil. anarebecasoares @ yahoo.com.br.
} 


\section{Introdução}

A insuficiência Cardíaca (IC) é a deficiência do coração em ofertar oxigênio e suprir a demanda metabólica tissular, sendo, portanto, uma entidade que possui uma grande repercussão orgânica (Volker et al., 2017). Tal magnitude também é expressa nas estatísticas epidemiológicas, um estudo realizado em 2010 revelou que 31\% dos pacientes internados e maiores de 70 anos, possuíam insuficiência cardíaca (Goldraich, et al., 2010). Nesse contexto, é possível observar que, além de ter uma repercussão sistêmica por hipóxia tissular, a insuficiência cardíaca possui alta prevalência na população idosa, que pelas condições do próprio envelhecimento (sarcopenia, redução do volume expiratório final pulmonar e etc) já possui baixa reserva funcional, o que implica em complicações cardíacas mais severas, bem como maior incremento na mortalidade nesta faixa etária e agravamento da gama de problemas de saúde pública (Moutinho et al., 2008).

No que se refere à própria fisiopatologia e classificação da Insuficiência Cardíaca, temos que esta se subdivide em varias formas, sendo a classificação mais importante àquela segundo o desempenho cardiovascular expresso pela Fração de Ejeção (FE), sendo classificadas em Insuficiência Cardíaca de Fração de Ejeção Preservada (ICFEP) aquelas que o desempenho cardíaco possui uma FE> 50\% e Insuficiência Cardíaca de Fração de Ejeção Reduzida (ICFER) aquelas que o desempenho cardíaco possui um FE $<40 \%$ (Volker et al., 2017). A terapêutica também difere com esta subdivisão da IC e é sabido que apenas na ICFER existem fármacos (Inibidores da Enzima Conversora da Angiotensina, Betabloqueadores e Antagonistas da Aldosterona) com comprovação solida de redução da mortalidade, fazendo que a ICFEP ainda careça de estudos de aprimoramento para redução de mortalidade (Moutinho et al., 2008).

A ICFEP é uma subdivisão da IC que possui com principal fator de risco para o seu desenvolvimento a Hipertensão Arterial Sistêmica (HAS), entidade bastante comum no nosso meio, principalmente pelo estilo de vida da modernidade, ao passo que a ICFER possui outros fatores de risco de peso quase paritário em seu desenvolvimento, como doença de chagas, doença valvar e doença cardíaca isquêmica (Estany e Garcia, 2016).

Partindo desta condição epidemiológica, é possível entender que o aumento da Resistencia Vascular Periférica(RVP) na HAS faz com que o miocárdio necessite de maior vigor para manter a FE suprindo a demanda metabólica tissular e o faz à custa de um aumento da massa miocárdica expressa em sua maioria pela Hipertrofia do Ventrículo 
Esquerdo (HVE) do tipo concêntrica (Volker et al., 2017). A HVE concêntrica impõe uma redução do lumem ventricular, fazendo com que o retorno venoso seja dificultado e se aprisione na circulação pulmonar, implicando sinais e sintomas respiratórios como dispneia paroxística noturna, estertoração e até mesmo chegando a causar o dramático quadro de edema agudo pulmonar o que faz com que a presença de pelo menos dois desses fatores já caracterize a IC e com grande possibildade de se caracterizar pelo contexto epidemiológico de incidência da HAS uma ICFEP (Goldraich et al., 2010).

Sendo assim, a ICFEP é uma entidade que pode ser prevenida por meio do tratamento adequado da HAS que se impõe na modernidade em duas vertentes, farmacológico e de medidas gerais (Goldraich et al., 2010).

Obviamente, é imprescindível a associação dos dois métodos terapêuticos, porem nunca a presença de apenas um deles deve ser abordagem única ao tratamento. Destaca-se entre a terapêutica de medidas gerais o estímulo ao exercício físico, que de forma extraordinária reduz os níveis tensionais e com auxílio farmacológico põe a RVP a números considerados menos deletérios ao organismo (Bocci, 2012).

No entanto, ainda permanece uma questão na cardiologia, não se sabe com clareza se a redução dos níveis tensionais auxilia de forma considerável na redução da massa miocárdica, base do desenvolvimento da Insuficiência cardíaca com fração de ejeção preservada (ICFEP), fazendo com que o presente estudo seja necessário e tenha como objetivo buscar, nas duas mais citadas bases de dados médicos, os efeitos da prática de atividade física no miocárdio de pacientes com IC, focando na ICFEP (Volker et al., 2017).

\section{Metodologia}

A presente revisão integrativa da literatura foi realizada nas bases de dados MEDLINE/PubMed e SciELO utilizando como palavras-chave PHYSICAL ACTIVITY e HEART FAILURE intercaladas pelo operador boleano AND a procura de estudos científicos que obtivessem no título ou no assunto as palavras-chave. Foram encontrados 1056 artigos na base MEDLINE/PubMed e 33 artigos na base SciELO, aplicando ainda o filtro de pesquisa temporal dos últimos cinco anos foram expressos 473 artigos na base MEDLINE/PubMed e 8 artigos na base SciELO, destes, resultou 311 estudos naquela e 8 nesta base de pesquisa quando se selecionou apenas estudos em humanos. A fase final 
consistiu na implicação de 41 artigos da MEDLINE/PubMed e 2 da base de dados SciElo após exclusão de artigos pelo titulo por não contemplarem a temática, em seguida, prosseguiu-se a leitura dos abstracts dos estudos e foram aplicados os critérios de inclusão previamente delimitados artigos com temática central envolvendo Insuficiência cardíaca, remodelamento cardíaco, atividade física e com população de estudo sendo indivíduos adultos, foram excluídos da pesquisa artigos com tema central voltado para população pediátrica, insuficiência cardíaca aguda ou estilo de vida sedentário. Ao final foram selecionados quatro artigos.

Na tabela 1 a seguir, a distribuição dos artigos utilizados neste estudo.

Tabela 1 - Distribuição dos artigos que fizeram parte do presente estudo.

\begin{tabular}{c|c|c|c|l}
\hline ARTIGO & AUTOR & ANO & ESTUDO & RESULTADOS \\
\hline $\begin{array}{c}\text { Molecular } \\
\text { Effects of } \\
\text { Novel }\end{array}$ & Volker et al & 2017 & $\begin{array}{c}\text { Revisão } \\
\text { Integrativa }\end{array}$ & $\begin{array}{l}\text { O programa de } \\
\text { exercícios físicos } \\
\text { invertem o } \\
\text { remodelamento } \\
\text { Training } \\
\text { Protocols }\end{array}$ \\
cardíaco.
\end{tabular}

Fonte: Dados da Pesquisa, 2019. 


\section{Resultados e Discussão}

A IC é uma das principais doenças cardiovasculares no Brasil, é sabido que os níveis tensionais elevados e, o estilo de vida sedentário é a base para o desenvolvimento de HAS que em longo prazo implicará no aumento da incidência de casos de IC e por consequência na prevalência da doença no País (Estany e Garcia, 2016).

A prática da atividade física reduz os níveis tensionais e faz parte da terapêutica da HAS, porem, quando se trata da orientação da pratica de atividade física em pacientes com IC, esta se faz de forma cautelosa principalmente naqueles com sintomas exuberantes classificados como Estágio III pela New York Heart Association (NYHA) por possuírem limitação a pratica de exercícios mais extenuantes que aumentam a demanda metabólica de oxigênio tissular além do que o coração possa suprir (Hedge e Solomon, 2017). No entanto, a prática de exercícios físicos com bom acompanhamento desencadeia uma serie de reações moleculares e liberação de substâncias endógenas e neuromediadores que reduzem a RVP ao ponto de reduzir o trabalho miocárdico (Volker et al., 2017).

Com essa redução do trabalho miocárdico, em portadores de ICFEP detentores de HVE concêntrica, a massa excessiva miocárdica adquirida por longos anos de altos níveis tensionais, os quais necessitavam ser vencidos pelo coração para adequada oferta de oxigênio aos tecidos, se reduz de forma paulatina (Haack e Zucker, 2015).

$\mathrm{Na}$ Tabela 1, estão elencadas quatro revisões integrativas de literatura realizadas nos últimos cinco anos de estudos que provam a redução da massa miocárdica, após programa de exercícios físicos de caráter não extenuante e com acompanhamento por profissionais treinados, em pacientes portadores de ICFEP.

Segundo os achados de Volker et al. (2017), na metanálise de Chen et al. (1994) é possível especular que a melhora da função endotelial por meio da liberação de vasodilatadores durante o exercício físico aeróbico repercutem na redução da massa miocárdica quando a pratica de exercícios físicos é feita de forma continua diária, por no mínimo 30 minutos e com foco em atividades aeróbicas que fornecem maiores níveis de oxigênio aos tecidos ao passo que diminui o trabalho cardíaco em suprir a grande exigência metabólica tissular. Tal estudo é corroborado pelos outros resultados de Volker et al. (2017), como o estudo de Hambrecht et al. (2004)que traz a evidência de aumento do volume diastólico final e aumento da câmara cardíaca esquerda hipertrofiada após a 
indução de um programa de exercícios aeróbicos em portadores de IC em um período de doze meses.

Na revisão integrativa de Hedge e Solomon (2017) existe uma redução paradoxal da massa miocárdica em portadores de ICFEP em função do aumento progressivo da intensidade aeróbica dos exercícios físicos por sete meses, sem causar extenuação física após cada sessão de 1 hora de exercício, o que nos faz inferir que a é necessário à procura do limite funcional do paciente de forma com que se progrida o nível de exercícios para uma redução da massa miocárdica até determinado estágio.

Quanto aos mediadores químicos é possível observar nos quatro estudos a incidência do oxido nítrico como principal vasodilatador mediado pelos exercícios. Tal vasodilatador exibe uma potência sustentável após a prática aeróbica que dura cerca de 2 horas, o que permite a redução da resistência vascular periférica e menor trabalho cardíaco para em longo prazo reduzir a massa miocárdica hipertrofiada.

\section{Conclusões}

Os presentes estudos tornam mais esclarecida a duvida sobre a redução da massa miocárdica em portadores de IC com programas de exercícios físicos, é notória a presença de benefícios em portadores de ICFEP que possuem como fisiopatologia a HVE concêntrica, sendo estes os principais beneficiados pela pratica de atividade aeróbica unida ao controle da pressão arterial, como já é bem empregado na terapêutica da IC na modernidade. No entanto, ainda se faz preciso mais estudos para tornar evidente os principais exercícios aeróbicos e o limite em que tais práticas reduzem a massa de um miocárdio hipertrofiado.

\section{Referências}

Bocchi, E. A. et al. Sociedade Brasileira de Cardiologia. Atualização da Diretriz Brasileira de Insuficiencia Cardiaca Crônica - 2012. Arq Bras Cardiol. 2012;98(supl1):1-13.

Chen HI, Li HT, Chen CC. Physical conditioning decreases norepinephrine-induced vasoconstriction in rabbits: possible roles of norepinephrine-evoked endothelium-derived relaxing factor. Circulation. 1994;90(2):970- 
Estancy, Eduardo Rivas; García, Susana Hernández. Entrenamiento físico en la insuficiencia cardíaca crónica: fisiopatología y evolución clínica. / [Physical training in chronic heart failure: pathophysiology and clinical evolution]. Medwave ; 16 Suppl 4: e6517, 2016 Sep 05.

Golbraich, L et al. Preditores Clinicos de Fração de Ejeção de Ventriculo Esquerdo Preservada na Insuficiencia Cardiaca Descompensada. Arq Bras Cardiol. 2010;94(3):36471.

Haack and Zucker. Central Mechanism For Exercice Training-Induced Redution in Sympatho-Excitation in Chronic Heart Failure. NIH Public Acess. Auton Neurosci. 2015 March ; 0: 44-50. doi:10.1016/j.autneu.2014.10.015.

Hambrecht R, Walther C, Möbius-Winkler S, Gielen S, Linke A, Conradi K, Erbs S, Kluge R, Kendziorra K, Sabri O, Sick P, Schuler G. Percutaneous coronary angioplasty compared with exercise training in patients with stable coronary artery disease: a randomized trial. Circulation. 2004 Mar 23;109(11):1371-8. Epub 2004 Mar 8.

Hegde and Solomon. Influence of Physical Activity on Hypertension and Cardiac Structure and Function. HHS Public Acess. Published in final edited form as: Curr Hypertens Rep. 2015 October ; 17(10): 77. doi:10.1007/s11906-015-0588-3.

Moutinho, MA et al. Heart Failure with Preserved Ejection Fraction and Systolic Dysfunction in the Community. Arq Bras Cardiol. 2008; 90(2):132-7.

Volker, Adams et al. Molecular effects of exercise training in patients with cardiovascular disease: focus on skeletal muscle, endothelium, and myocardium. Am J Physiol Heart Circ Physiol 313: H72-H88, 2017. First published May 5, 2017; doi:10.1152/ajpheart.00470.2016.

\section{How to cite this article (APA format):}

Bandeira, Luiz Arthur Bevilaqua; Silva, Talina Carla da; Silva, Macerlane Lira; Assis, Elisangela Vilar de; Vasconcelos, Melina Maria Loiola Melo; Oliveira, Ana Rebeca Soares Maia de. (2019). Preditores de Insuficiência Cardíaca na População Adulta. Am. In. Mult. J., October. (7) 4, 111117.

Received: 10/12/2019

Accepted: 10/20/2019 